\title{
Estrutura populacional e aspectos reprodutivos de Corydoras aeneus (Siluriformes, Callichthyidae) em riachos da bacia do rio Ivinhema, Alto rio Paraná
}

\author{
Fabiane S. Ferreira' ${ }^{1,2} \&$ Yzel R. Súarez²
}

\author{
1. Programa de Pós-Graduação em Recursos Naturais, Universidade Estadual de Mato Grosso do Sul/UEMS, 79804-970, Dourados, MS, Brasil. \\ (fabianesfbio@gmail.com) \\ 2. Universidade Estadual do Mato Grosso do Sul/UEMS, Centro de Análise e Monitoramento Ambiental/CINAM, Laboratório de Ecologia. Rod. Dourados-ltahum \\ km 12, 79804-970, Dourados, MS, Brasil. (yzel@uems.br)
}

\begin{abstract}
Population structure and reproductive aspects of Corydoras aeneus (Siluriformes, Callichthyidae) in streams of the River Ivinhema Basin, Upper Paraná River. Despite of increase of studies about biology of small fish species, the knowledge of basic aspects of biology great portion of species still is incipient. Thereat, the aim of this study was analyze the population structure and the reproductive aspects of Corydoras aeneus (Gill, 1858) in streams of the Ivinhema River Basin, Upper Paraná River. The samplings were performed from 2008 to 2011 at fifty seven sites distributed in basin. They were analyzed 684 individuals, including 213 females, 190 males and 281 obtained only the dada of standard length. The maximum standard length registered for analyzed population was $50.19 \mathrm{~mm}$ for females and $45.24 \mathrm{~mm}$ for males, so females were larger than males. The sex ratio was $52.7 \%$ females and of $47.1 \%$ males, no significant variation $\left(X^{2}=1.313 ; \mathrm{p}=0.252\right)$ between sex. How much the Lengthweight relationship both sexes presented isometric growth. The highest proportion of mature females was observed in from October to December, although were observed mature females in others months of year. It was observed that $50 \%$ of females were sexually mature at $33.54 \mathrm{~mm}$. Mean fecundity was estimated $359.47 \mathrm{eggs}$, and presented total spawning. The recruitment pattern no presented a unimodal period, although the more intensity was observed from October to December. The asymptotic length was estimated at $54.59 \mathrm{~mm}$ and growth rate at 1.1 year ${ }^{-1}$. The growth performance index was estimated to 3.51 , the longevity at 2.72 years and the mortality rate at 1.48 year $^{-1}$.
\end{abstract}

KEYWORDS. Length-weight relationship, fecundity, mortality, sex-ratio.

RESUMO. Apesar do crescente número de estudos sobre biologia de espécies de peixes de pequeno porte, o conhecimento dos aspectos básicos da biologia da maioria das espécies ainda é incipiente. Com isso, o objetivo desse estudo foi descrever alguns aspectos populacionais e reprodutivos de Corydoras aeneus (Gill, 1858) em riachos da bacia do rio Ivinhema, Alto rio Paraná. As amostragens foram realizadas entre 2008 e 2011 em 57 riachos distribuídos na bacia. Foram analisados 684 indivíduos, sendo 213 fêmeas e 190 machos, além de 281 exemplares analisados apenas quanto aos dados de comprimento padrão. O comprimento padrão máximo da população analisada foi de $50,19 \mathrm{~mm}$ para as fêmeas e de 45,24 mm para machos; Desta forma, as fêmeas foram maiores que os machos. A proporção sexual foi de $52,7 \%$ para fêmeas e de $47,1 \%$ para os machos, sem variação significativa entre os sexos $\left(X^{2}=1,313 ; \mathrm{p}=0,252\right)$. Quanto à relação peso/comprimento ambos os sexos apresentaram a crescimento isométrico. As maiores frequências de fêmeas maduras foram observadas nos meses de outubro a dezembro, embora tenham sido observadas fêmeas maduras em outros meses do ano. Estimou-se que 50\% das fêmeas estão sexualmente maduras com 33,54 mm de comprimento padrão. A fecundidade média foi estimada em 359,47 oócitos, sendo que a população apresenta desova total. O padrão de recrutamento não apresentou um padrão unimodal, embora a maior intensidade tenha sido observada nos meses de outubro a dezembro. O comprimento assintótico foi estimado em $54,59 \mathrm{~mm}$ e a taxa de crescimento de 1,1 ano $^{-1}$. O índice de performance de crescimento foi estimado em 3,51; a longevidade de 2,72 anos e a mortalidade de 1,48 ano ${ }^{-1}$.

PALAVRAS-CHAVE. Relação peso-comprimento, fecundidade, mortalidade, proporção sexual.

A ictiofauna neotropical é uma das mais diversificadas do mundo. Contudo, o conhecimento da biologia básica de suas espécies ainda é um dos maiores desafios da ictiologia (LOWE-MCCONNELL, 1999), sendo que as espécies de pequeno porte são as menos conhecidas, desde o ponto de vista taxonômico até a quantificação de aspectos da ecologia populacional (SANNA-KAISA \& JUKKA, 2004).

A reprodução é fundamental para a manutenção das populações e seu estudo, consequentemente, contribui para o estabelecimento de medidas de conservação e manejo das espécies. Dessa forma, o conhecimento de aspectos da estrutura populacional, tais como proporção sexual, mortalidade, comprimento assintótico, primeira maturação, padrão de recrutamento, fecundidade, período reprodutivo, longevidade, entre outros, são parâmetros de história de vida essenciais para avaliar os aspectos reprodutivos e são indicativos do crescimento da população, bem como permitem a compreensão sobre a dinâmica populacional das espécies de peixes e as respostas resultantes a alterações antrópicas (LOWE-MCCONNELL, 1999).

Os Siluriformes, junto com os Characiformes, constituem as ordens mais representativas de peixes da Região Neotropical. Compreendem, em geral, formas de hábitos bentônicos e noturnos, mas muitas são ativas durante o dia, principalmente em águas turvas (BRITSKI et al., 2007).

Corydoras Lacepède, 1803 (Callichthyidae, Siluriformes) é composto por 170 espécies (EsCHMEYER, 2013) amplamente distribuídas na América do Sul, ocorrendo em uma variedade de habitats (BRITTO et al., 2007). De acordo com ReIs (2003), em média, duas novas espécies de Corydoras têm sido descritas ao ano durante as últimas décadas. No Alto rio Paraná, seis espécies foram 
registradas até então (LANGEANI et al., 2007); no entanto, nos rios da porção sul-matogrossense da bacia, apenas C. aeneus foi registrada até então (SÚAREZ et al., 2011).

Existe uma série de estudos quanto a aspectos reprodutivos de espécies de Corydoras (LoIR et al., 1989; ARANHa et al., 1993; Bervian \& Fontoura, 1994; KoHDa et al., 1995; 2002; PAXTON, 1997; PrUZsinsZKY \& Ladich, 1998; KaAtz \& Lobel, 1999; Alkins-Koo, 2000; HuYSENTRUYT \& AdRIAENS (2005); MAZZOLDi et al., 2007; RONDINELI \& BRAGA, 2009; MAHAPATRA \& DUTTA, 2014). No entanto, informações sobre $C$. aeneus na região estudada ainda são escassas.

Existem estudos sugerindo que $C$. aeneus possa ser utilizado como indicador de degradação ambiental, como resultado de sua adaptação à respiração aérea, o que leva ao incremento em sua abundância relativa em riachos pobres em oxigênio dissolvido (CASATTI et al., 2006). Neste sentido, o presente estudo buscou fornecer informações sobre alguns aspectos populacionais e reprodutivos de C. aeneus na bacia do rio Ivinhema, Alto rio Paraná, respondendo às seguintes questões: (1) como se estrutura a população de $C$. aeneus, por classes de tamanho? (2) qual a proporção sexual desta população? (3) existe diferença na relação peso-comprimento entre os sexos? (4) qual o período reprodutivo da espécie? (5) existe diferença no fator de condição entre os sexos? (6) qual o tamanho de primeira maturação das fêmeas? (7) qual a fecundidade média e relativa da espécie? (8) existe correlação da fecundidade com o comprimento e com o peso das fêmeas? (9) quais os parâmetros básicos de crescimento, mortalidade e o padrão de recrutamento da espécie analisada?

\section{MATERIAL E MÉTODOS}

Área de estudo. A bacia do rio Ivinhema está localizada à margem direita do rio Paraná, no Mato Grosso do Sul, Brasil (Fig. 1). Possui cerca de $600 \mathrm{~km}$ de extensão e $45.000 \mathrm{~km}^{2}$ de área, sendo um dos poucos remanescentes com características lóticas do Alto Paraná (sem influência grandes represamentos). A nascente do rio Ivinhema está localizado a aproximadamente $700 \mathrm{~m}$ de altitude na nascente do rio Dourados, que flui para o rio Brilhante e, com a sua fusão com o rio Vacaria, é renomeado para Ivinhema (SúAREZ et al., 2011).

Coleta de dados e procedimentos laboratoriais. Os peixes foram coletados em 57 pontos amostrais distribuídos ao longo de córregos da bacia do rio Ivinhema, no período de janeiro de 2008 a fevereiro de 2011. Cada um desses pontos apresentava cerca de 100 metros de extensão. As amostragens, autorizadas pelo IBAMA (SISBIO $\mathrm{n}$. 13458-1), foram realizadas no período diurno, utilizandose uma peneira retangular medindo $1,2 \times 0,8 \mathrm{~m}(2 \mathrm{~mm}$ de abertura de malha), bem como redes de arrasto $(1,5 \times 5 \mathrm{~m})$, também com $2 \mathrm{~mm}$ de abertura de malha. Em campo, os peixes foram fixados em formol a $10 \%$ e, em laboratório, preservados em álcool etílico $70 \%$. A identificação das espécies foi realizada com base em GraÇA \& PaVAnelli
(2007). Exemplares-testemunho encontram-se depositados na Coleção de Peixes do NUPELIA (UEM-NUP 9362).

Foi analisado um total de 684 exemplares e, para cada exemplar, foi registrado o comprimento padrão (CP; em milímetros, $0,1 \mathrm{~mm}$ de precisão) e o peso total (PT; em gramas, com precisão de $0,001 \mathrm{~g}$ ). Do total de exemplares, 403 indivíduos foram dissecados para observação macroscópica das gônadas, identificação do sexo, obtenção do peso das gônadas (PG) e, posteriormente, para a análise da fecundidade.

Os estágios de maturação gonadal foram definidos segundo a escala modificada de VAzzoler (1996). Para fêmeas, foram identificados os seguintes estágios: (1) imaturo (ovários translúcidos e pequenos), (2) em maturação (ovários com oócitos opacos e pequenos ou médios), (3) maduros (ovários que ocupam completamente a cavidade celomática, apresentando oócitos com grandes dimensões e coloração amarelada), (4) esgotado (ovários com aspecto flácido e com hemorrágico) e (5) repouso (ovários que estavam em processo de recuperação após a desova). Os estágios de maturação das gônadas dos machos foram definidos como: (1) imaturo (testículos translúcidos e pequenos), (2) em maturação (testículos translúcidos, no entanto mais volumosos e com aspecto granular) e (3) maduros (testículos volumosos e branqueados).

Quinze fêmeas maduras (estágio 3) foram selecionadas aleatoriamente para obtenção da fecundidade absoluta. Essas gônadas foram colocadas em solução de Gilson para total dissociação dos oócitos. Destas, foram contados todos os oócitos vitelogênicos e medidos 150 oócitos em diferentes fases de desenvolvimento, escolhidos aleatoriamente, para determinação do tipo de desova.

Análise de dados. Os exemplares fazem parte de várias amostragens na bacia do rio Ivinhema, realizadas em diferentes períodos. Dessa forma, a influência interanual foi desconsiderada, optando-se em agrupar por trimestres, sendo que os meses de janeiro a março foram considerados como primeiro trimestre, abril a junho como segundo, julho a setembro como terceiro e outubro a dezembro como o quarto trimestre; assim os exemplares analisados em cada trimestre foram agrupados ao longo dos anos amostrados.

A proporção sexual foi calculada por meio do teste de qui-quadrado, com correção de Yates (ZAR, 1996), visando verificar possíveis variações entre os sexos e a variação temporal ao longo dos trimestres.

A relação entre o peso e comprimento padrão dos peixes foi calculada separadamente para machos e fêmeas, através de regressão não linear, por meio da equação alométrica $y=a x^{b}$, sendo $y$ o peso, $x$ o comprimento e $a$ e $b$ constantes estimadas pelo método dos mínimos quadrados (SANTOS, 1978). A comparação dos valores estimados do coeficiente angular de crescimento (b) entre machos e fêmeas foi realizada através da comparação dos valores de seus intervalos de confiança.

A partir dos valores do peso total de cada exemplar (PT) e do peso das gônadas (PG), foi estimado o índice Gonadossomático (IGS) ao longo dos trimestres analisados, 


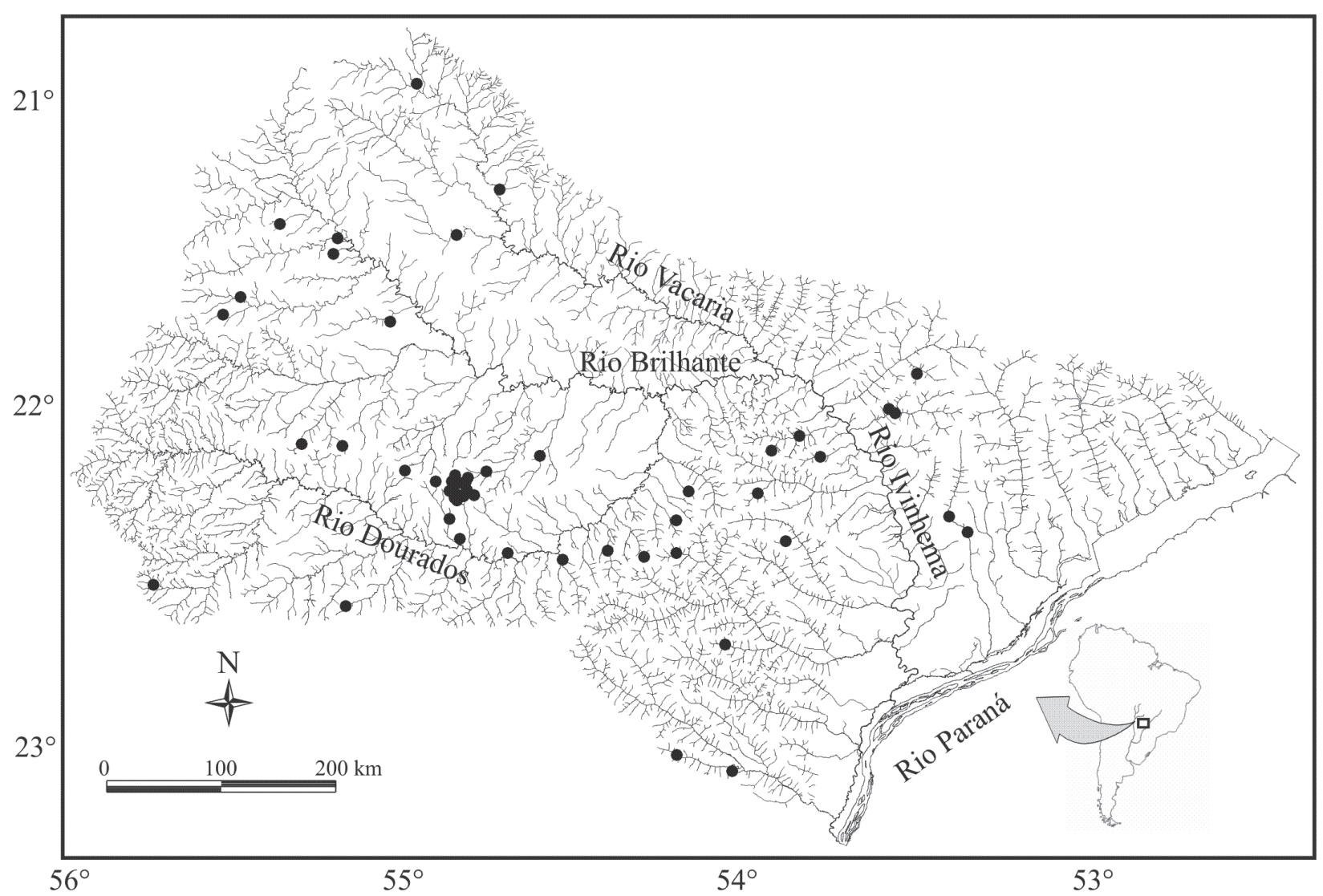

Fig. 1. Localização dos locais amostrados na bacia do rio Ivinhema, Alto rio Paraná, Mato Grosso do Sul, Brasil.

por meio da equação IGS=PG/PT*100 (VAZZOLER, 1996). Sendo assim, para determinação do período reprodutivo, foi utilizada a frequência dos estágios de maturação gonadal e a variação dos valores médios do IGS, definindo o período de maior atividade reprodutiva, sendo que, no primeiro caso, utilizou-se o teste qui-quadrado e, para o IGS, foi utilizado o teste não paramétrico de Kruskall-Wallis, buscando verificar se a mediana do IGS varia ao longo dos trimestres.

Calculou-se o fator de condição alométrico como um indicador de bem-estar de cada indivíduo, refletindo condições alimentares recentes, dado pela relação entre o peso e o comprimento do indivíduo, sendo expresso por: $\mathrm{K}$ $=\mathrm{PT} / \mathrm{CP}^{\mathrm{b}}$, onde $\mathrm{PT}=$ peso total $; \mathrm{CP}=$ comprimento padrão e $b=$ coeficiente angular de regressão entre PT/CP (BRAGA, 1986). Com o objetivo de verificar se o fator de condição dos peixes varia ao longo dos trimestres e, considerando que não foi constatada normalidade nos dados (ZAR, 2010), realizou-se um teste não paramétrico de Kruskall-Wallis para ambos os sexos, aplicando-se o teste a posteriori de Nemenyi-Damico-Wolfe-Dunn.

O tamanho de primeira maturação foi obtido da seguinte forma: após a análise do estágio de maturação gonadal, fêmeas imaturas foram consideradas como não reprodutivas (zero) e as demais como reprodutivas (um). Desta forma, uma análise de regressão logística foi aplicada para quantificar a mudança na probabilidade de uma fêmea se reproduzir em função de seu comprimento padrão (VAZZOLER, 1996).
A fecundidade absoluta obtida de cada fêmea foi correlacionada com seu comprimento padrão $(\mathrm{CP})$ através da correlação de Spearman, visando verificar se existe uma relação do comprimento padrão e a fecundidade.

O comprimento assintótico foi estimado a partir do maior indivíduo capturado, utilizando-se a equação de PAuLY (1983): $L \infty=L \max / 0,95$. O valor estimado da taxa de crescimento (k) foi obtido utilizando-se o método ELEFAN I (Eletronic Lengths-Frequency Analysis) (PAULY \& DAVID, 1981), através da função de crescimento de Von Bertalanffy: $\mathrm{L}(\mathrm{t})=\mathrm{L} \infty(1-\exp [-\mathrm{k}(\mathrm{t}-\mathrm{to})])$, onde: $\mathrm{L} \infty=$ Comprimento assintótico, $\mathrm{k}=$ taxa de crescimento $\left(\mathrm{ano}^{-1}\right), \mathrm{t} 0=$ idade nominal quando o comprimento é zero, $\mathrm{t}=$ idade (meses/ ano) (BertalanfFy, 1938; Gulland, 1977; SANTOs, 1978). O método ELEFAN I está inserido no programa FISAT (FAO-ICLARM Stock Assessment Tools) (GAYANILO et al., 1996; Gayanilo \& Pauly, 1997), que busca as melhores combinações na estimativa dos parâmetros, em função dos melhores ajustes ou maiores valores de Rn ("goodness of fit index").

A mortalidade total $(Z)$, definida como igual à mortalidade natural (M), foi obtida segundo a fórmula empírica de PAuly (1980), que utiliza a informação dos parâmetros de crescimento (L $\infty$ e k) e a temperatura média $\left({ }^{\circ} \mathrm{C}\right)$ do ambiente em que a espécie foi coletada, seguindo a equação: $\ln M=-0,0152-0,279 \ln L \infty+0,6543 \ln k+$ $0,463 \ln T^{\circ} \mathrm{C}$. A longevidade foi calculada pelo valor estimado segundo a equação proposta por TAYLOR (1958): $t \max =t_{0}+$ 
$2,996 / k$, sendo $t_{0}=0$, e, pela observação do número de coortes ( 1 coorte $=1$ ano) geradas pelo modelo de Von Bertalanffy realizado anteriormente. O FISAT ainda forneceu o índice de performance de crescimento $(\varphi)$ que foi obtido através da equação proposta por PAULY \& MUNRO (1984): $\phi=\log k+$ $2 \log L \infty$. O índice de performance de crescimento fornece informações para possível comparaçãodos parâmetros estimadoscom parâmetros de outras espécies ou grupos filogeneticamente relacionados, devendo apresentar valores próximos de três e semelhantes entre espécies aparentadas, como indicativo de que os parâmetros populacionais foram estimados de forma correta.

O padrão de recrutamento foi obtido por meio da distribuição de frequência mensal de comprimento padrão e dos parâmetros de crescimento (L $\infty$ e k) estimados anteriormente, utilizando a rotina incluída no FISAT (Gayanilo \& Pauly, 1997).

\section{RESULTADOS}

Foram analisados 684 indivíduos de C. aeneus, sendo 403 utilizados para determinação do período reprodutivo (213 fêmeas e 190 machos). Constatou-se que as fêmeas atingiram maior comprimento padrão $(50,19 \mathrm{~mm} ;$ média $=38,8 \pm \mathrm{dp}=6,8)$ e maior peso $(6,35$ g; média $=3,0 \pm d p=1,3)$ que os machos $(45,24 \mathrm{~mm}$; comprimento médio $=35,1 \pm \mathrm{dp}=4,4$ e peso $3,89 \mathrm{~g}$; média $=2,07 \pm \mathrm{dp}=0,7$ ).

Através do teste qui-quadrado (Fig. 2), não foi constatada diferença significativa na proporção sexual total entre os sexos $\left(X^{2}=1,313 ; \mathrm{p}=0,252\right)$. Essas proporções sexuais também não diferiram significativamente ao longo dos trimestres $\left(X^{2}=6,227 ; \mathrm{p}=0,101\right)$, entretanto, no terceiro (julho a setembro) apresentou uma diferença significativa

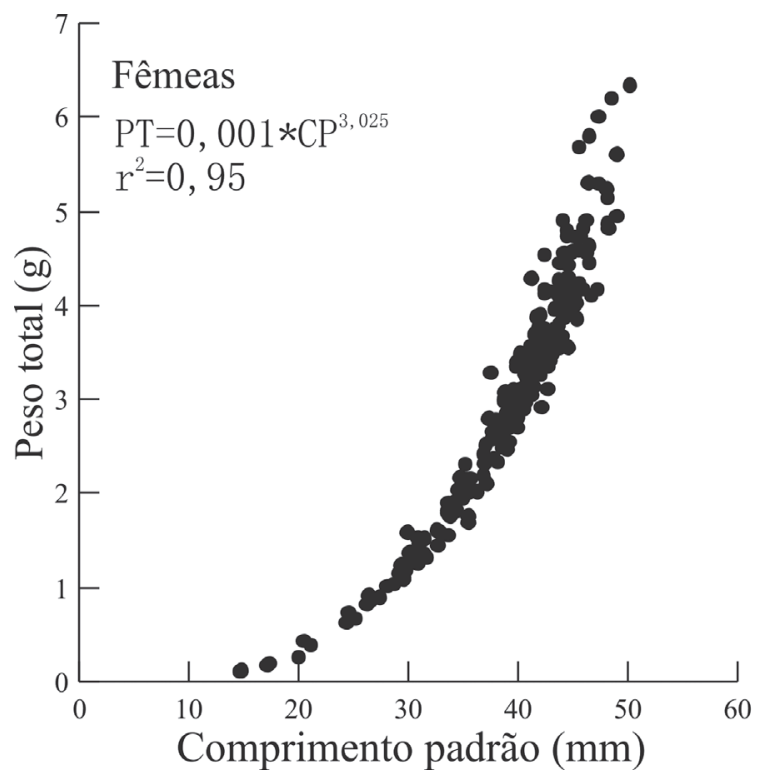

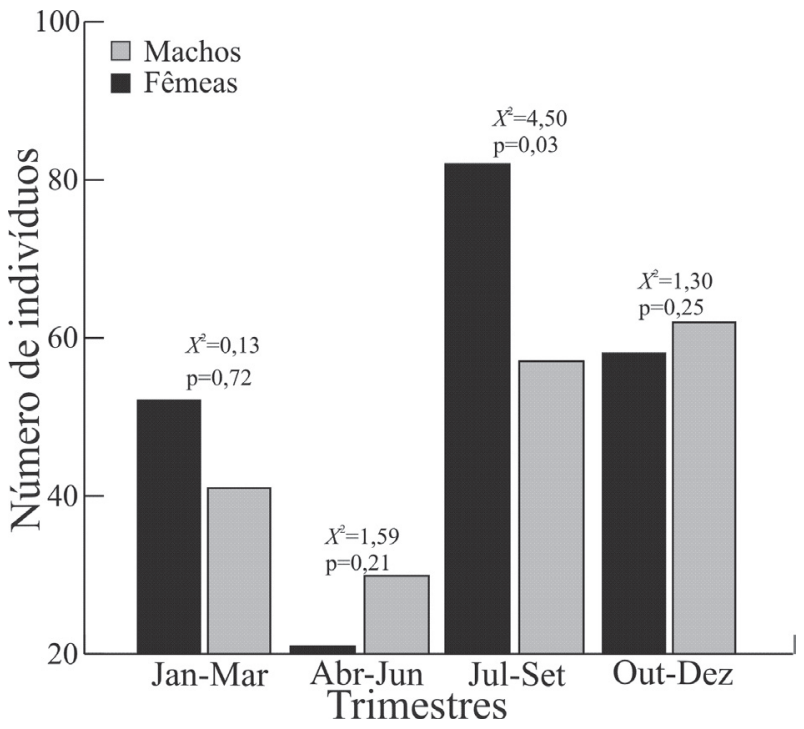

Fig. 2. Número de indivíduos por sexo de Corydoras aeneus (Gill, 1858) analisados por trimestres, na bacia do rio Ivinhema, Alto rio Paraná, Mato Grosso do Sul, Brasil.

$\left(X^{2}=4,50 ; \mathrm{p}=0,03\right)$. Dessa forma, mesmo ocorrendo uma variação entre os sexos nos meses de julho a agosto, a proporção sexual não diferiu da proporção esperada de 1:1.

A relação peso/comprimento (RPC) (Fig. 3) resultou nas expressões $\mathrm{PT}=0,001 * \mathrm{CP}^{2,958}\left(\mathrm{r}^{2}=0,94\right)$, com intervalo de confiança entre 2,833 e 3,083 para os machos, e PT $=0,001 * \mathrm{CP}^{3,025}\left(\mathrm{r}^{2}=0,95\right)$ para as fêmeas, com intervalo de confiança entre 2,895 e 3,156. Portanto, ambos os sexos apresentam crescimento isométrico, ou seja, aumentam o peso proporcionalmente ao comprimento ao longo da sua ontogenia.

Os valores do Índice gonadossomático (IGS) para machos de foram menores do que para as fêmeas (Fig. 4).

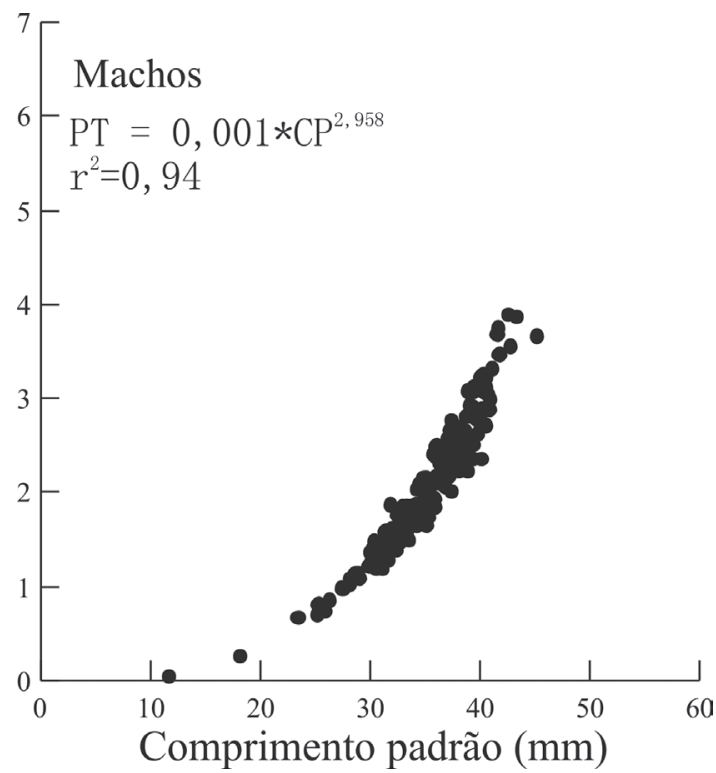

Fig. 3. Relação Peso total/Comprimento padrão para 213 fêmeas e 190 machos de Corydoras aeneus (Gill, 1858) amostrados na bacia do rio Ivinhema, Alto rio Paraná, Mato Grosso do Sul, Brasil. 
Os maiores valores medianos do IGS ocorreram no quarto trimestre para ambos os sexos. Através do teste de Kruskal Wallis, foi constatada uma variação significativa nos valores medianos do IGS das fêmeas ao longo dos trimestres analisados $(\mathrm{KW}=25,147 ; \mathrm{p}=0,001)$, ocorrendo diferenças significativas no terceiro e quarto trimestre. No entanto, o mesmo resultado não ocorreu para os machos, não sendo constatada nenhuma variação nos valores medianos do IGS (KW=7,511; $\mathrm{p}=0,057)$. Dessa forma, apenas as fêmeas apresentaram variação na atividade reprodutiva ao longo dos trimestres analisados.

Quanto aos estágios de maturação gonadal (Fig. 5), observou-se maior proporção de fêmeas $(58,6 \%)$ e machos $(54,8 \%)$ maduros no quarto trimestre. Este resultado é consistente com o resultado do IGS, sugerindo que a maior intensidade reprodutiva da população estudada ocorra nos meses de outubro a dezembro, ainda que indivíduos maduros tenham ocorrido durante todo o ano.

Os valores médios do fator de condição (Fig. 6) não apresentaram variação significativa ao longo dos trimestres analisados, nem para machos ( $\mathrm{KW}=4,066$; $\mathrm{p}=0,254)$, nem para fêmeas $(\mathrm{KW}=6,952 ; \mathrm{p}=0,073)$. Dessa forma, os maiores valores do fator de condição para fêmeas e machos ocorreram no terceiro e no quarto trimestre, respectivamente.

Estimou-se que $50 \%$ das fêmeas atingem a maturidade sexual com 33,54 $\mathrm{mm}\left(\mathrm{IC}_{0.05}=32,4\right.$ a 34,5$) \mathrm{e}$ $100 \%$ a atingem com $44,11 \mathrm{~mm}\left(\mathrm{IC}_{0,05}=41,51 \mathrm{a} 49,21\right)$. Para os machos, estimou-se o $\mathrm{L}_{50}$ em $33,15 \mathrm{~mm}\left(\mathrm{IC}_{0,05}=32,0\right.$ a 34,02) e o $\mathrm{L}_{100} \operatorname{com} 49,61 \mathrm{~mm}\left(\mathrm{IC}_{0,05}=45,89\right.$ a 56,68).

A fecundidade das 15 fêmeas de C. aeneus analisadas variou entre 73 e 778 , com média estimada em 359,47 oócitos ( $\pm \mathrm{dp}=178,66)$, com uma correlação significativa entre a fecundidade e o comprimento padrão (Spearman $\mathrm{r}=0,55 ; \mathrm{p}=0,04$ ) (Fig. 8).

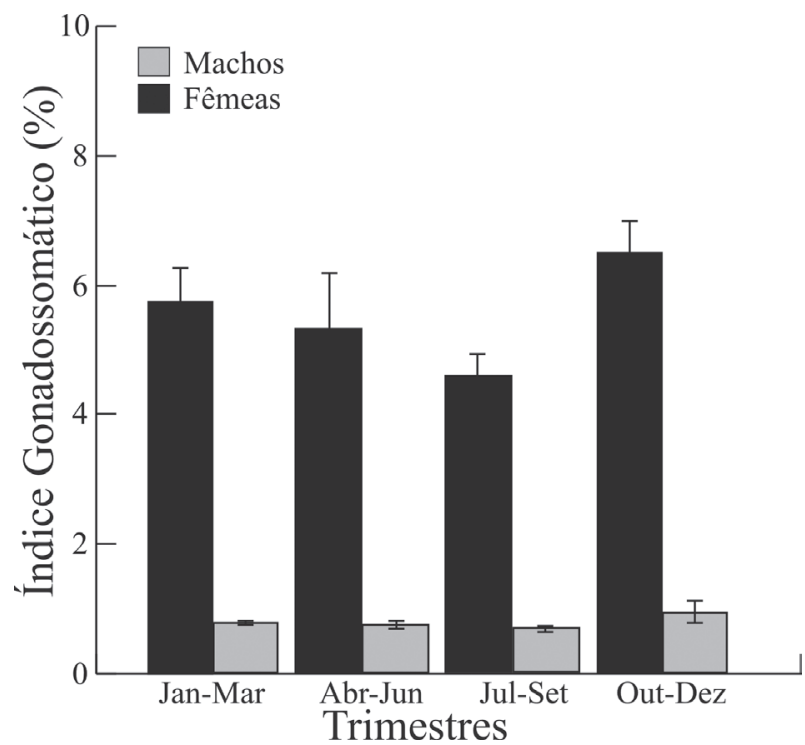

Fig. 4. Variação trimestral dos valores do Índice Gonadossomático - IGS de 213 fêmeas e 190 machos de Corydoras aeneus (Gill, 1858) amostrados na bacia do rio Ivinhema, Alto rio Paraná, Mato Grosso do Sul, Brasil.

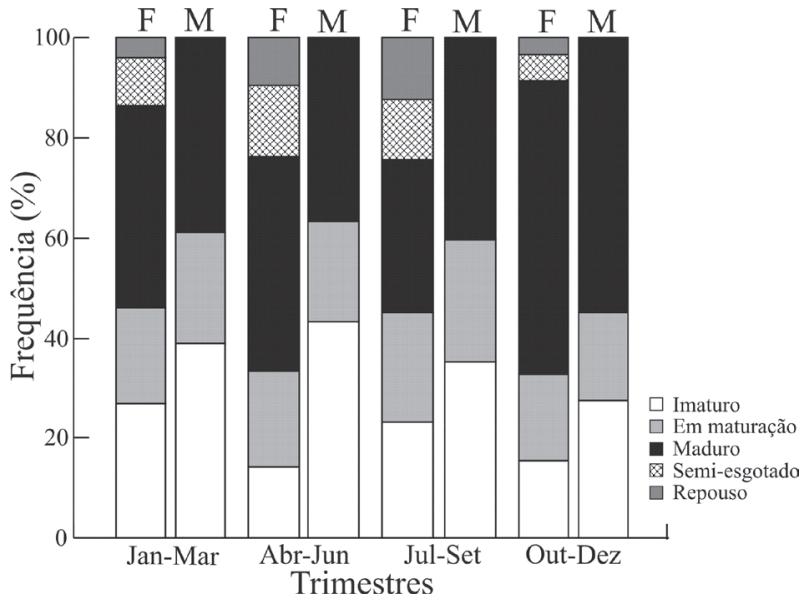

Fig. 5. Frequência trimestral dos estágios de maturação para 213 fêmeas e 190 machos de Corydoras aeneus (Gill, 1858) amostrados na bacia do rio Ivinhema, Alto rio Paraná, Mato Grosso do Sul, Brasil.

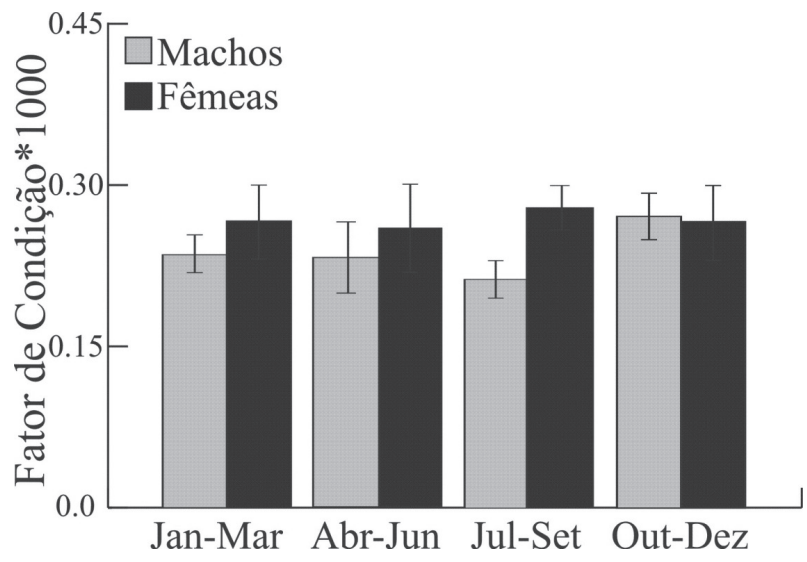

Fig. 6. Média ( \pm Desvio Padrão) do Fator de condição $(* 1000)$ por trimestres para 213 fêmeas e 190 machos de Corydoras aeneus (Gill, 1858) amostrados na bacia do rio Ivinhema, Alto rio Paraná, Mato Grosso do Sul, Brasil.

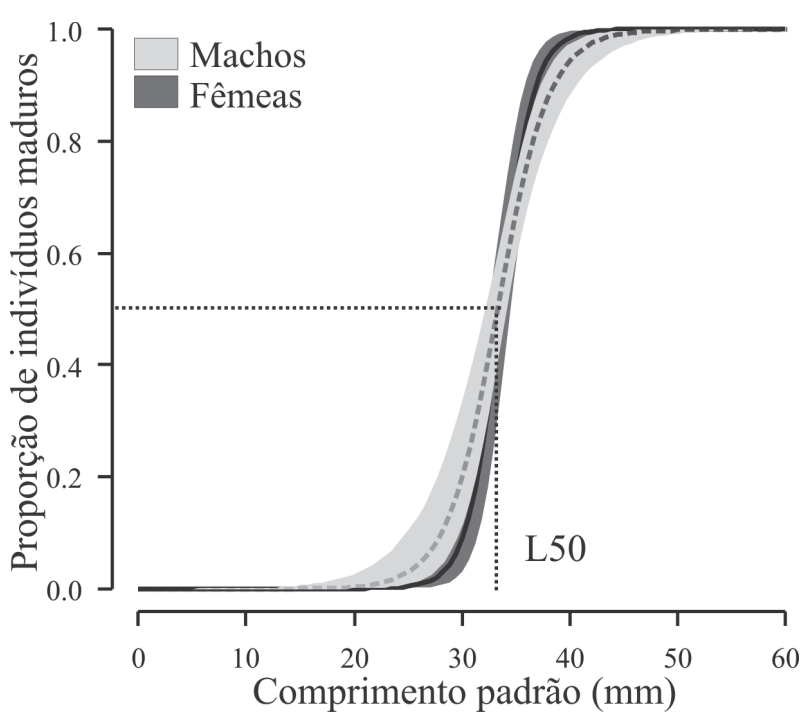

Fig. 7. Proporção da maturação sexual para 213 fêmeas e 190 machos em função do comprimento padrão de Corydoras aeneus (Gill, 1858) amostrados na bacia do rio Ivinhema, Alto rio Paraná, Mato Grosso do Sul, Brasil. 


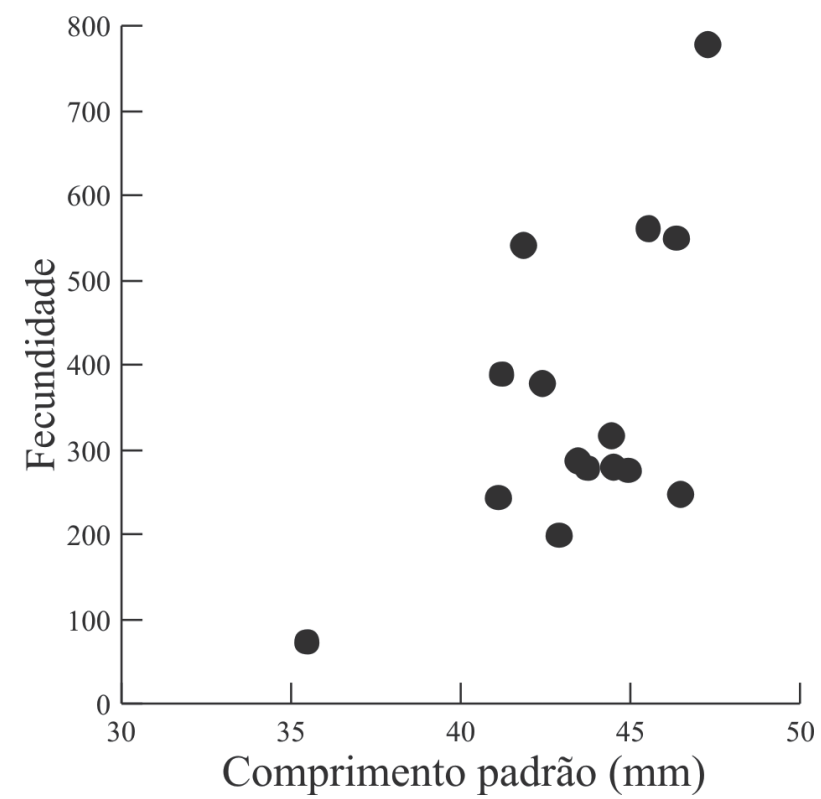

Fig. 8. Relação entre a fecundidade total para em função do comprimento padrão de 15 fêmeas de Corydoras aeneus (Gill, 1858) amostradas na bacia do rio Ivinhema, Alto rio Paraná, Mato Grosso do Sul, Brasil.

A distribuição da frequência absoluta dos diâmetros dos oócitos vitelogênicos apresentou dois picos, demonstrando que a espécie apresenta um processo de desenvolvimento sincrônico em dois grupos, sugerindo uma desova do tipo total (Fig. 9). Foi observada alta frequência de oócitos de reserva, que possivelmente apresentarão desenvolvimento sincrônico até chegarem à maturidade e serem eliminados no próximo período de desova. A amplitude de variação dos oócitos vitelogênicos variou de 0,15 e $2,03 \mathrm{~mm}$.

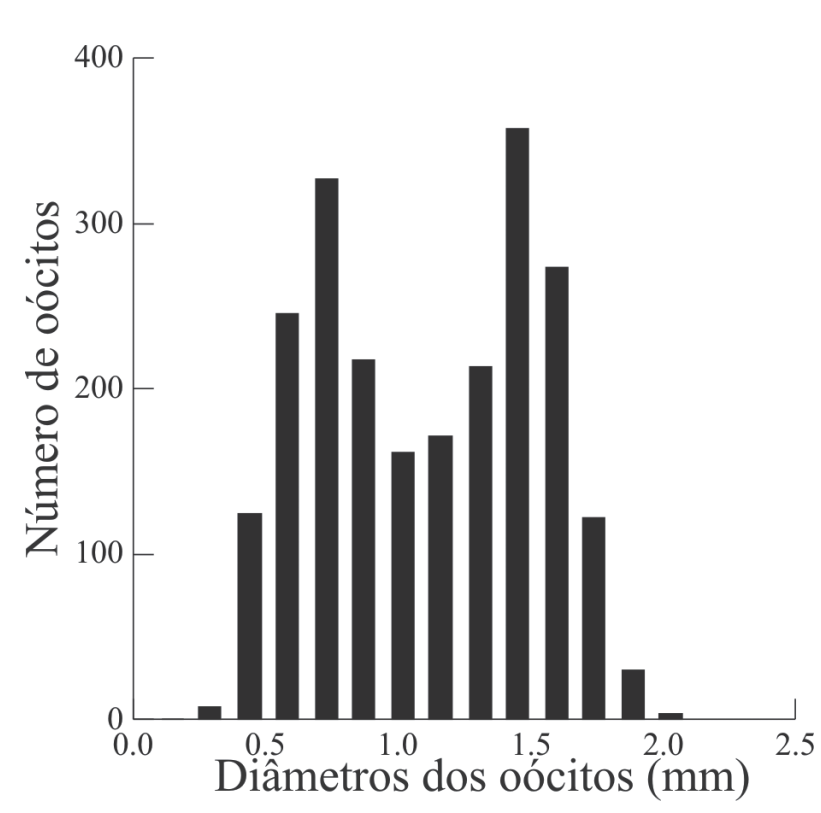

Fig. 9. Distribuição de frequência do número de oócitos por classes de diâmetro para 15 fêmeas de Corydoras aeneus (Gill, 1858) amostradas na bacia do rio Ivinhema, Alto rio Paraná, Mato Grosso do Sul, Brasil.
Os maiores picos de recrutamento para machos e fêmeas ocorreram no terceiro trimestre (Fig. 10), coincidindo com os maiores valores médios do fator de condição para os machos. Dessa forma, o padrão do recrutamento foi consistente para a população analisada, devido à ocorrência de indivíduos maduros em todos os trimestres.

Estimou-se o comprimento assintótico em 54,59 $\mathrm{mm}$, enquanto a mortalidade natural (Z) ficou em 1,48 ano $^{-1}$ e a taxa de crescimento (k) em $1,1 \mathrm{ano}^{-1}$, com longevidade estimada em 2,72 anos (Fig.11). O índice de performance de crescimento $(\varphi)$ foi estimado em 3,51.

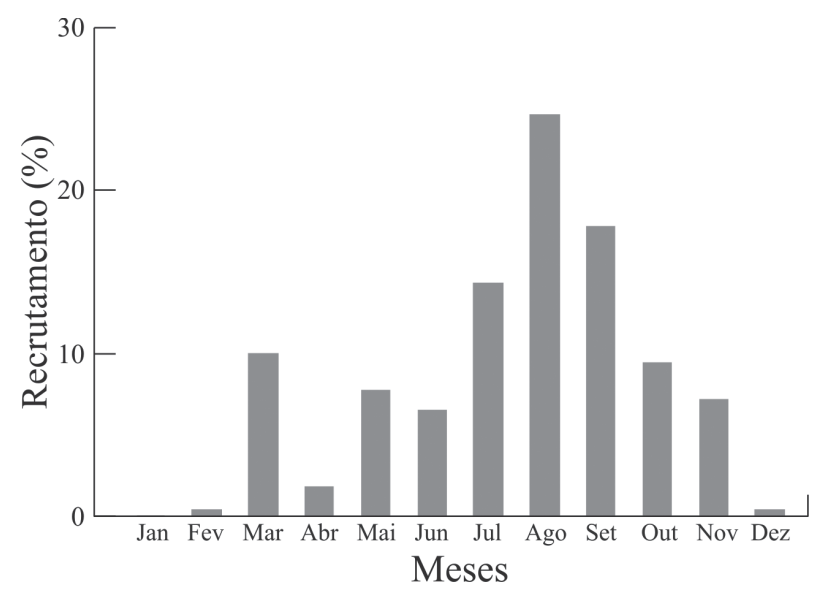

Fig. 10. Frequência de recrutamento ao longo dos meses para Corydoras aeneus (Gill, 1858) amostrados na bacia do rio Ivinhema, Alto rio Paraná, Mato Grosso do Sul, Brasil.

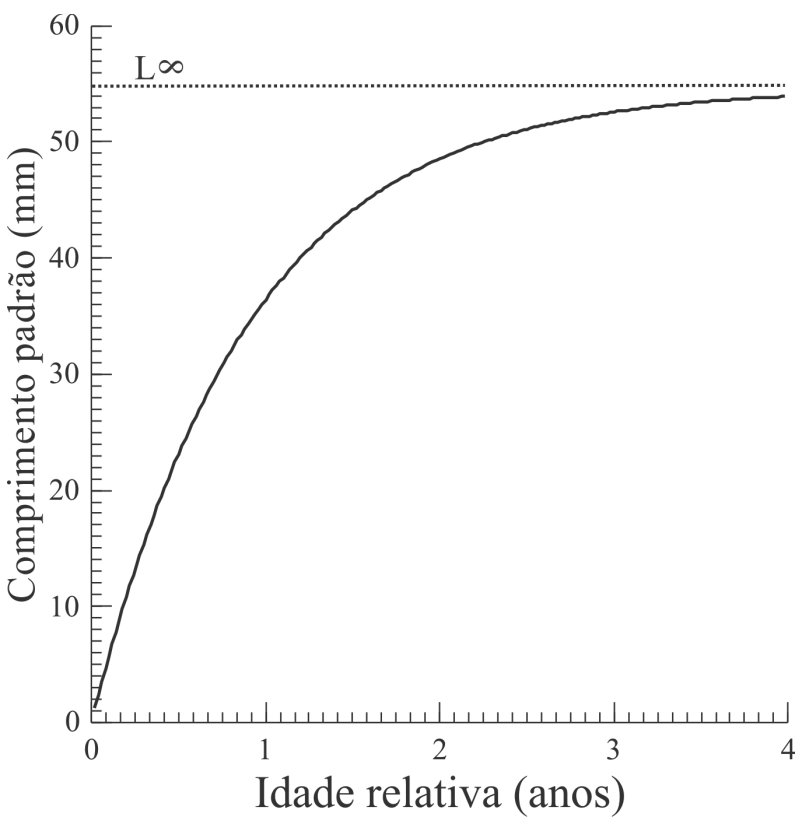

Fig. 11. Curva de crescimento método de Von Bertalanffy para Corydoras aeneus (Gill, 1858) amostrados na bacia do rio Ivinhema, Alto rio Paraná, Mato Grosso do Sul, Brasil. 


\section{DISCUSSÃO}

A proporção sexual de 1:1 verificada em C. aeneus é normalmente observada também em muitas espécies de peixes (VAZzolER, 1996). Resultados similares foram constatados para os calictídeos Corydoras flaveolus Ihering, 1911 (Rondinel \& BRAGA, 2009) e Corydoras paleatus Jenyns, 1842 (Bervian \& Fontoura, 1994). No entanto, estudos realizados com outras populações de $C$. aeneus (ARANHA et al., 1993; AlKins-Koo, 2000) e com populações de Corydoras carlae Nijssen \& Isbrücker, 1983 (ARANHA et al., 1993) e Aspidoras fuscoguttatus Nijssen \& Isbrücker, 1976 (ARAúJo \& GARUTTI, 2002), a proporção sexual diferiu entre de 1:1.

Diversos fatores podem interferir na determinação da proporção sexual nos peixes (eg. crescimento, mortalidade e comportamento), atuando de forma diferenciada sobre os sexos e alterando a proporção sexual (NASCIMENTO et $a l ., 2012$ ) entre espécies ou em uma mesma população de um ano para o outro (NiKOLSKY, 1963; RoNDINELI \& Braga, 2009). Na maioria dos estudos, observa-se uma proporção sexual de 1:1 para a população como um todo, podendo ser constatadas alterações indicando, por exemplo, o predomínio de machos ou fêmeas em diferentes classes de comprimento ou em diferentes épocas (VAZZOLER, 1996). Dessa forma, a predominância de um sexo é uma informação importante para a caracterização da estrutura de uma espécie ou população, além de fornecer subsídios para estudo de outros aspectos como a avaliação do potencial reprodutivo e estimativas do tamanho do estoque (VAzZOLER, 1996).

As fêmeas de $C$. aeneus foram maiores que os machos, resultado que é consistente com os encontrados para outras espécies de Callichthyidae (BERvian \& Fontoura, 1994; PruzsinszKY \& LADICH, 1998; AlKinsKoo, 2000; AraúJo \& GARUTTI, 2002; KoHDa et al., 2002; MAZzoldi et al., 2007; Rondineli \& BRAGA, 2009). O dimorfismo sexual é frequentemente registrado entre os Characiformes e Siluriformes (LOWE-MCCONNELL, 1999), uma vez que o custo da reprodução para as fêmeas é maior do que para os machos em decorrência do maior gasto energético, por necessitarem comportar grandes ovários para garantir maior fecundidade. Dessa forma, as fêmeas apresentam maior peso principalmente no período que antecede o período da desova (VAzzoler et al., 1989).

A relação peso/comprimento (RPC) é um importante parâmetro utilizado em estudos de populações de peixes e sua aplicação se baseia na estimativa do peso de um indivíduo com base no seu comprimento, podendo variar de uma espécie para outra, entre sexos e entre populações (Le-Cren, 1951; Rondineli \& BraGa, 2009). Além disso, as alterações corporais estão associadas às mudanças fisiológicas que ocorrem em função do ciclo reprodutivo da espécie (Rondineli \& BraGA, 2009). As fêmeas e os machos de $C$. aeneus apresentaram um crescimento isométrico e equivalente, tanto em peso quanto em comprimento. Outros calictídeos, como A. fuscoguttatus
(ARaúJo \& Garutti, 2002) e C. flaveolus (Rondineli \& BRAGA, 2009) apresentaram, respectivamente, crescimento alométrico positivo e alométrico negativo. Em peixes, o peso e comprimento são parâmetros que podem ser influenciados de acordo com a disponibilidade de alimento, período reprodutivo da espécie e fatores abióticos de cada ambiente (eg. temperatura e salinidade) (TESCH, 1971; WeAtherley \& Gill, 1987; Moutopoulos \& Stergiou, 2002).

Os maiores valores do IGS foram encontrados nos meses de outubro a dezembro para ambos os sexos, indicando o período de reprodução da população estudada. A frequência dos estágios de maturação gonadal também indicou a atividade reprodutiva mais acentuada nesse período, contudo, também apresentaram machos e fêmeas maduras ao longo de todo ano. Alkins-Koo (2000) constatou um período reprodutivo semelhante para $C$. aeneus, assim como RonDineli \& BRAGA (2009) para C. flaveolus. Aranha et al. (1993) observaram fêmeas maduras de $C$. carlae durante todo o ano, enquanto $C$. aeneus apresentou fêmeas maduras no período de março a junho. Bervian \& Fontoura (1994), ao estudarem a dinâmica populacional de $C$. paleatus, constataram que a reprodução da espécie ocorreu durante o ano todo.

Os resultados do presente trabalho sugerem que, na bacia do rio Ivinhema, as condições ambientais são favoráveis à reprodução de $C$. aeneus ao longo de todo o ano. Segundo Winemiller (1989) e Braga et al. (2008), espécies de pequeno porte, tolerantes a ambientes com frequentes distúrbios, podem apresentar repetitivos episódios reprodutivos, podendo estar associados à adaptação e à alta capacidade de recolonização destes habitats. Considerando que outras espécies do grupo (e.g. C. carlae, C. flaveolus, A. fuscogutattus) também apresentam período reprodutivo longo, esta pode ser uma estratégia comum às espécies da subfamília Corydoradinae.

A idade ou tamanho da primeira maturação gonadal é uma tática reprodutiva muito instável, relacionada com o crescimento e a interações do genótipo-ambiente (WoOtTON, 1992; VAZzoler et al., 1997). As fêmeas e os machos de C. aeneus alcançaram a primeira maturação gonadal com comprimento diferente, sugerindo que existe uma diferença entre as taxas de crescimento e desenvolvimento entre os sexos, evidente principalmente pelo maior comprimento atingido pelas fêmeas. Comprimento maior na primeira maturação indica maiores investimentos em fecundidade, uma vez que fêmeas maiores têm maior quantidade de oócitos (WoOtTON, 1992). ARANHA et al. (1993) registrou $30 \mathrm{~mm}$ como sendo o comprimento padrão do menor indivíduo maduro de C. carlae e C. aeneus, enquanto BERVIAN \& FONTOURA (1994) observaram maior frequência de fêmeas reprodutivas de C. paleatus entre 45 e $50 \mathrm{~mm}$ e ARAúJo \& GARUTTI (2002) encontraram um tamanho médio da primeira maturação para A. fuscoguttatus de 37,1 $\mathrm{mm}$ para fêmeas e $30,5 \mathrm{~mm}$ para os machos. Ainda que a metodologia utilizada em cada trabalho seja distinta, os dados encontrados no presente estudo são similares aos 
existentes na literatura. $\mathrm{O}$ frequente uso de $C$. aeneus em aquariofilia reforça a necessidade de que estas informações sejam obtidas de forma a subsidiar a possível exploração comercial deste estoque, determinando-se um tamanho mínimo de captura.

O fator de condição da população analisada sugere que as reservas nutritivas acumuladas ao longo do ano pela espécie foram utilizadas entre os meses de abril e junho (quando o fator de condição é menor), para o desenvolvimento oócitário nos meses de julho a setembro (quando o fator de condição é maior), preparando-se para a reprodução durante os meses de outubro a dezembro (maior valor de IGS). O fator de condição pode variar intra- e interespecificamente, dependendo das características do ambiente (Le-Cren, 1951; Rondineli \& Braga, 2009), do período do ano e do desenvolvimento ontogenético de cada indivíduo (Froese, 2006; Camara et al., 2011), podendo ser afetado pela maturação gonadal, sexo e dieta (Tesch, 1971; Oscoz et al., 2005).

A população analisada apresentou a RPC equivalente entre os sexos, no entanto, o comprimento máximo alcançado foi maior para as fêmeas. Esse resultado, aliado ao comprimento de primeira maturação e à proporção sexual geral equivalente entre os sexos, sugere um ajuste nestas características de história de vida, ao menos para a população avaliada. O maior tamanho alcançado pelas fêmeas tem sido amplamente explicado como um mecanismo para permitir o aumento da fecundidade individual (VAzzoler et al., 1989; Wootton, 1992). Ajustes nas características de história de vida têm sido discutidos há várias décadas em ecologia evolutiva, como mecanismos de evitar a competição sexual entre machos e fêmeas. Dessa forma, uma proporção sexual equivalente, associada os tamanhos de primeira maturação ajustados e taxas de crescimento equivalentes, permitiriam que ambos os sexos tivessem as mesmas probabilidades de deixar descendentes (FISHER, 1930).

A fecundidade é uma característica individual e está adaptada às condições do ciclo de vida da espécie, variando com o crescimento, densidade populacional, disponibilidade de alimento e taxa de mortalidade (NiKOLSKY, 1969). Para Corydoradinae, informações sobre a fecundidade foram assinaladas para Aspidoras menezesi Nijssen \& Isbrücker,1976 (50 a 60 oócitos), Corydoras paleatus (250 a 400 oócitos), Corydoras hastatus Eigenmann \& Eigenmann, 1888 (30 a 60 oócitos), Corydoras pygmaeus (20 a 40 oócitos) (Burgess, 1989), C. aeneus (média 171 oócitos) (MaHAPATRa \& DutTA, 2014); todas essas espécies são utilizadas em aquariofilia, sendo que essas informações foram obtidas em condições artificiais, as quais podem ter interferido nos resultados.

Por outro lado, em condições naturais, C. aeneus apresentou fecundidade máxima com comprimento padrão de 47,3 mm (778 oócitos). Corydoras paleatus mostrou fecundidade máxima com comprimento padrão de 55,0 mm (450 a 500 oócitos) (Bervian \& Fontoura, 1994), $C$. aeneus a revelou com um comprimento padrão de $60 \mathrm{~mm}$
(1.046 oócitos) (Alkins-Koo, 2000); A. fuscoguttatus com um comprimento total máximo de 37,5 a 46,8 mm (51 a 166 oócitos) (ARAúJo \& GaRUTTI, 2002). Dessa forma, os resultados sugerem que a fecundidade em peixes é variável de acordo com as condições ambientais, temporais e em função do comprimento e o peso (BraGa et al., 2007).

A desova total de $C$. aeneus no presente estudo difere dos resultados constatados por AlKINS-Koo (2000), Araújo \& Garutti (2002) e Rondinelli \& Braga (2009), que constataram desova parcelada em C. aeneus, A. fuscogutattus e C. flaveolus, respectivamente. O tipo de desova pode ter inúmeras táticas que integram as estratégias reprodutivas (VAZZOLER, 1996), podendo ser moldado pelo ambiente, determinando a sobrevivência das populações (WINEMILLER, 1989), onde a desova total está relacionada com período reprodutivo curto, ao contrário da desova parcelada (NiKolsky, 1963). No entanto, para C. aeneus, foram verificados vários episódios reprodutivos ao longo do ano, sendo que, em cada episódio, apenas um lote de oócitos é eliminado, promovendo maior dissipação. Deste modo, os resultados reforçam o padrão contínuo do recrutamento observado para a população analisada.

Os oócitos maduros de $C$. aeneus apresentaram diâmetro máximo de $1,45 \mathrm{~mm}$, enquanto os maiores diâmetros de oócitos em $A$. fuscoguttatus foi de 1,75 (Araújo \& Garutti, 2002) e em C. flaveolus de 2,39 mm (RoNDINELLI \& BRAGA, 2009). O tamanho do oócitos é uma das estratégias reprodutivas mais importantes para os peixes ósseos (VAZZOLER, 1996), associado com a disponibilidade de alimento que a larva poderá explorar e também com a probabilidade de sobrevivência dos juvenis (BAGENAL, 1971), ou seja, oócitos maiores favorecem a eclosão de larvas maiores e mais aptas a explorar o ambiente (BRAGA et al., 2007). Essa estratégia pode compensar a redução da fecundidade (Wootton, 1992). Espécies que produzem oócitos menores e mais suscetíveis à predação, normalmente necessitam ter fecundidade mais alta (WоOtTON, 1992).

Os parâmetros de crescimento e mortalidade podem conduzir diferentes características e estratégias de história de vida de espécies de peixes (NIKOLSKY, 1969), influenciados pela variação das condições ambientais, tais como a disponibilidade de alimentos, temperatura e densidade populacional (Lowe-McConNelL, 1999). Além disso, pode ocorrer variação dos parâmetros populacionais de mesma espécie em ambientes diferentes (WoOtTON, 1992; BENITEZ \& Súarez, 2009). Na bacia do rio Ivinhema, C. aeneus apresentou alta taxa de crescimento e mortalidade natural. A alta taxa de crescimento usualmente esta relacionada à intensa predação que os indivíduos sofrem desde o momento de eclosão dos ovos (Lowe-MCConNeLL, 1999); com isso, os indivíduos crescem rapidamente, alcançando a maturidade mais cedo (BLANCK \& LAMOUROUX, 2007) e apresentam comprimentos assintóticos menores do que aquelas espécies em que a taxa de crescimento é menor, normalmente representadas por indivíduos de grande porte (LizAma \& Ambrósio, 2003).

Dessa forma, indivíduos que crescem rápido e 
amadurecem mais cedo, têm uma longevidade maior e uma fecundidade elevada, sugerindo que esta estratégia seja o resultado da adaptação em habitats com altas temperaturas e que favorecem altas taxas metabólicas (BEVERTON \& Holt, 1959). Embora a maioria dos peixes ocorra em ambientes com maior taxa de oxigênio, algumas espécies são adaptadas para sobreviver em habitats com baixa concentração de oxigênio (hipóxia) porque possuem características morfológicas e fisiológicas que permitem a respiração direta a partir do oxigênio atmosférico (JoBLING, 1994). Com isso, em habitats hipóxicos as espécies apresentam alta fecundidade (BLANCK et al., 2007) e oócitos menores que não demandam maiores taxas de oxigênio para atender a sua demanda metabólica (KroGH, 1959).

Em síntese, $C$. aeneus exibiu vários episódios reprodutivos ao longo de todo o ano, uma vez que apenas um lote de oócitos é eliminado por episódio reprodutivo e alta fecundidade. A população analisada revelou altas taxas de crescimento e mortalidade. Como C. aeneus ocorre em habitats sujeitos a intensas variações na qualidade da água, a estratégia oportunista da espécie pode ser considerada uma forma de se ajustar à imprevisibilidade do ambiente.

Agradecimentos. À Gabriela S. V. Duarte, Karina K. Tondato, Lucilene F. Vieira e Maiane J. Pereira, pelo auxílio no laboratório, e Marcelo M. Souza, Olemar C. Martins, João Paulo da Silva, Wagner Vicentin, Lilian Paula Vasconcelos e Madson S. Melo, pela ajuda nas amostragens. À UEMS, CNPq e FUNDECT, pelo apoio financeiro.

\section{REFERÊNCIAS BIBLIOGRÁFICAS}

Alkins-Koo, M. 2000. Reproductive timing of fishes in a tropical intermittent stream. Environmental Biology of Fishes 57:49-66.

Aranha, R. J. M. R.; Caramaschi, E. P. \& Caramaschi, U. 1993. Ocupação espacial, alimentação e época reprodutiva de duas espécies de Corydoras Lacépède (Siluroidei, Callichthyidae) coexistentes no rio Alambari (Botucatu, São Paulo). Revista Brasileira de Zoologia 10(3):453-466.

AraúJo, R. B. \& GarutTi, V. 2002. Biologia reprodutiva de Aspidoras fuscoguttatus (Siluriformes, Callichthyidae) em riacho de cabeceira da bacia do Alto rio Paraná. Iheringia, Série Zoologia 92:89-98.

Bagenal, T. B. 1971. The interrelation of the size of fish eggs, the date of spawning and the production cycle. Journal of Fish Biology 3:207-219.

BENITEZ, R. S. \& SÚAREZ, Y. R. 2009. Biologia populacional de Serrapinus notomelas (Eingenmann, 1915) (Characiformes, Cheirodontinae) em um riacho de primeira ordem na bacia do rio Dourados, Alto Rio Paraná. Pan-American Journal of Aquatic Sciences 4(3):271-278.

BertalanfFy, L. vON.1938. A quantitative theory of organic growth (inquiries on growth laws. II). Human Biology 10:181-213.

Bervian, G. \& Fontoura, N. F. 1994. Dinâmica populacional da ictiofauna da lagoa Fortaleza, Cidreira, Rio Grande do Sul. III Corydoras paleatus (Jenyns, 1842) (Teleostei, Callichthyida). Biociências 2(2):15-23.

Beverton, R. H. J. \& Holt, S. J. 1959. A review of the lifespans and natural mortality rates of fish in nature and their relation to growth and other physiological characteristics. In: WolstenholmE, G. E. W. \& Connor, M. O. eds. CIBA Colloquium on Ageing. London, Churchill. v. 5, p.142-180.

Blanck, A. \& Lamouroux, N. 2007. Large-scale intraspecific variation in life-history traits of European freshwater fish. Journal of Biogeography 34:862-875.

Blanck, A.; Tedesco, P. A. \& Lamoueoux, N. 2007. Relationships between life-history strategies of European freshwater fish species and their habitat preferences. Freshwater Biology 52:843-859.
BRAGA, F. M. S. 1986. Estudo entre fator de condição e relação pesocomprimento para alguns peixes marinhos. Brazilian Journal of Biology 46(2):339-346.

Braga, F. M. S.; Gomiero, L. M. \& Souza, U. P. 2008. Aspectos da reprodução e alimentação de Neoplecostomus microps (Loricariidae, Neoplecostominae) na microbacia do Ribeirão Grande, serra da Mantiqueira oriental (Estado de São Paulo). Acta Scientiarum, Biological Sciences 30(4):455-463.

Braga, F. M. S.; Souza, U. P. \& Carmassi, A. L. 2007. Dinâmica populacional de Characidium lauroi e C. alipioi (Teleostei, Crenuchidae) na microbacia do Ribeirão Grande, serra da Mantiqueira Oriental, estado de São Paulo. Acta Scientiarum, Biological Sciences 29(3):281-287.

Britski, H. A.; Silimon, K. K. S. \& Lopes, B. S. 2007. Peixes do Pantanal, Manual de Identificação. Brasília, Embrapa. 227p.

Britto, M. R.; Lima, F. C. T \& Hidalgo, M. H. 2007. Corydoras ortegai, a new species of Corydoradine catfish from the lower rio Putumayo in Peru (Ostariophysi: Siluriformes: Callichthyidae). Neotropical Ichthyology 5(3):293-300.

Burgess, W. E. 1989. An atlas of freshwater and marine catfishes: a preliminary survey of the Siluriformes. Neptune, TFH. 784p.

CAmara, E. M.; Caramaschi, E. P. \& Petry, A. C. 2011. Fator de condição: bases conceituais, aplicações e perspectivas de uso em pesquisas ecológicas com peixes. Oecologia Australis 15(2):249-274.

Casatti, L.; Langeani, F.; Silva, A. M. \& Castro, R. M. 2006. Stream fishes, water and habitat quality in a pasture dominated basin, southeastern Brazil. Brazilian Journal of Biology 66(2B):681-699.

Eschmeyer, W. N. 2013. Catalog of Fishes. San Francisco, California Academy of Sciences. Disponível em $<$ http://research.calacademy. org/ichthyology/catalog>. Acesso em 20.06.2015.

FISHER, R. A. 1930. The genetical theory of natural selection. London, Oxford University Press. $318 \mathrm{p}$.

Froese, R. 2006. Cube law, condition factor and length-weight relationship: history, meta-analysis and recommendations. Journal of Applied Ichthyology 22:241-253.

Gayanilo, F. C. JR. \& Pauly, D. 1997. The FAO-ICLARM Stock Assessment Tools (FISAT) Reference manual. FAO Computerized Information Series (Fisheries) 8:1-196.

Gayanilo, F. C. JR.; Sparre, P. \& Pauly, D. 1996. FAO-IClarm Stock Assessment Tools. User's Guide. FAO computerized information series (Fisheries). 126p.

Graça, W. J. \& Pavanelli, C. S. 2007. Peixes da planície de inundação do Alto Rio Paraná e áreas adjacentes. Maringá, EDUEM. 241p.

Gulland, J. 1977. The management of marine fisheries. Seattle, University of Washington Press. 198p.

Huysentruyt, F. \& Adriaens, D. 2005. Adhesive structures in the eggs of Corydoras aeneus (Gill, 1858; Callichthyidae). Journal of Fish Biology 66:871-876.

Jobling, M. 1994. Fish Bioenergetics. London, Chapman and Hall. 326p.

KAATZ, I. M. \& LoBeL, P. S. 1999. Acoustic behaviour and reproduction in five species of Corydoras catfishes (Callichthyidae). The Biological Bulletin 197:241-242.

Kohda, M.; TanimuRa, M.; KiKUe-NaKamura, M. \& Yamagishi, S. 1995. Sperm drinking by female catfishes: a novel mode of insemination. Environmental Biology of Fishes 42:1-6.

Kohda, M.; Yonebayashi, K.; NaKamura, M.; Ohnishi, N.; Seki, S.; TAKAHASHI, D. \& TAKEYAMA, T. 2002. Male reproductive success in a promiscuous armoured catfish Corydoras aeneus (Callichthyidae). Environmental Biology of Fishes 63:281-287.

Krogh, A. 1959. The Comparative Physiology of Respiratory Mechanisms. Philadelphia, University of Pennsylvania Press. 172p.

Langeani, F.; Castro, R. M. C.; Oyakawa, O. T.; Shibatta, O. A.; Pavanelli, C. S. \& Casatti, L. 2007. Diversidade da ictiofauna do Alto Rio Paraná: composição atual e perspectivas futuras. Biota Neotropica 5(1):75-78.

Le-Cren, E. D. 1951. The lenght-weight relationship and seasonal cycle in gonad weight and condition in the perch (Perca fluviatilis). Journal of Animal Ecology 20(2):201-219.

Lizama, M. A. P. \& Ambrósio, A. M. 2003. Crescimento, recrutamento e mortalidade do pequi Moenkhausia intermedia (Osteichthyes, Characidae) na planície de inundação do alto rio Paraná, Brasil. Acta Scientiarum, Biological Sciences 25(2):328-333. 
Loir, M.; Cauty, C.; Planquete, P. \& LeBail, P. Y. 1989. Comparative study of the male reproductive tract in seven families of SouthAmerican catfishes. Aquatic Living Resources 2:45-56.

Lowe-McConNeLL, R. H. 1999. Estudos ecológicos de comunidades de peixes tropicais. São Paulo, EDUSP. 534p.

Mahapatra, B. K. \& DutTA, S. 2014. Breeeding and rearing of an exotic ornamental catfish, Corydorus aeneus (Gill, 1858) in Kolkata, West Bengal and its economics. Proceedings of the Zoological Society 68:159-163.

Mazzoldi, C.; LoRenzi, V. \& Rasotto, M. B. 2007. Variation of male reproductive apparatus in relation to fertilization modalities in the catfish families Auchenipteridae and Callichthyidae) (Teleostei: Siluriformes). Journal of Fish Biology 70:243-256.

Moutopoulos, D. K. \& Stergiou, K. I. 2002. Length-weight and lengthlength relationships of fish species from the Aegean Sea (Greece). Journal of Applied Ichthyology 18:200-203.

Nascimento, W. S.; Yamamoto, M. E. \& ChellapPa, S. 2012. Proporção sexual e relação peso-comprimento do peixe anual Hypsolebis antenori (Cyprinodontiformes: Rivulidae) de poças temporárias da região semiárida do Brasil. Biota Amazônica 2(1):37-44.

Nikolsky, G. V. 1963. The ecology of fishes. London, Academic Press. 352p.

. 1969. Theory of fish population dynamics. Edinburgh, Oliver \& Boyd. 323p.

Oscoz, J.; Campos, F. \& Escala, M. C. 2005. Weight-length relationships of some fish species of the Iberian Peninsula. Journal of Applied Ichthyology 21:73-74.

Pauly, D. 1980. On the interrelationships between natural mortality, growth parameters and mean environmental temperature in 175 fish stock. Internacional Council Exploration of the Sea 39:175-192.

1983. Some simple methods for the assessment of tropical fish stocks. FAO Fisheries Technical Paper 234:1-52.

Pauly, D. \& David, N. 1981. ELEFAN I, a BASIC program for the objective extraction of growth parameters from length frequencies data. Meeresforschung 28(4):205-211.

Pauly, D. \& MunRo, J. L. 1984. Once more on the comparison of growth in fish and invertebrates. ICLARM Fishbyte 2(1):1-21.

Paxton, C. G. M. 1997. Shoaling and activity levels in Corydoras. Journal of Fish Biology 51:496-502.

PruZsinsZKY, I. \& LADICH, F. 1998. Sound production and reproductive behaviour of the armoured catfish Corydoras paleatus (Callichthyidae). Environmental Biology of Fishes 53:183-191.
ReIs, R. E. 2003. Family Callichthyidae (Armored catfishes). In: ReIs, R. R.; Kullander, S. O. \& Ferraris, JR. C. J. eds. Check list of the freshwater fishes of South and Central America. Porto Alegre, EDIPUCRS. p.291-309.

Rondineli, G. R. \& Braga, F. M. S. 2009. Biologia populacional de Corydoras flaveolus (Siluriformes, Callichthyidae) no Rio Passa Cinco, sub-bacia do Rio Corumbataí, São Paulo, Brasil. Biota Neotropica 9(4):45-57.

SAnNA-KaISA, J. \& JuKKa, S. 2004. Sustentable use of ornamental fish populations in Peruvian Amazonia. Lyonia 7(2):53-59.

SANTOS, E. P. 1978. Dinâmica de populações aplicada à pesca e piscicultura. São Paulo, Hucitec. 129p.

Súarez, Y. R.; Souza, M. M.; Ferreira, F. S.; Pereira, M. J.; Silva, E. A.; Ximenes, L. Q. L.; Azevedo, L. G.; Martins, O. C. \& LimaJunIOR, S. E. 2011. Patterns of species richness and composition of fish assemblages in streams of the Ivinhema River basin, Upper Paraná River. Acta Limnologica Brasiliensia 23(2):177-188.

TAYLOR, C. C. 1958. Cod growth and temperature. Journal du Conseil International pourl'Exploration de la Mer 23:366-370.

Tesch, F. W. 1971. Age and growth. In: RicKer, E. eds. Methods for assessment of fish production in fresh waters. Oxford, Blackwell, p.99-130.

Vazzoler, A. E. A. M. 1996. Biologia da reprodução de peixes Teleósteos: Teoria e Prática. Maringá, EDUEM. 169p.

Vazzoler, A. E. A. M.; Amadio, S. A. \& Caraciollo-Malta, M. 1989. Aspectos biológicos de peixe amazônicos: XII. Indicadores quantitativos do período de desova das espécies do gênero Sema prochilodus (Characiformes, Prochilodontidae) do baixo Rio Negro, Amazonas. Brasil. Revista Brasileira de Biologia 49(1):165-173.

Vazzoler, A. E. A. M.; Suzuki, H. I.; Marques, E. E. \& Lizama, M. A. P. 1997. Primeira maturação gonadal, períodos e áreas de reprodução. In: VAzzoler, A. E. A. M.; Agostinho, A. A.; Hahn, N. S. eds. A planície de inundação do alto rio Paraná. Aspectos físicos, biológicos e socioeconômicos. Maringá, Eduem, p.249-266.

Weatherley, A. H. \& Gill, H. S. 1987. The Biology of Fish Growth. London, Academic Press. 443p.

WINEMILLER, K. O. 1989. Patterns of variation in life history among South American fishes in seasonal environments. Oecologia 81:225-241.

Wootton, R. J. 1992. Fish ecology. New York, Chapman and Hall. 212p.

ZAR, J. H. 1996. Biostatistical analysis. London, Prentice Hall. 662p. 2010. Biostatistical analysis. Upper Saddle River, Pearson Prentice Hall. 944p. 